\title{
Five-year delay would spell end of ITER
}

Osamu Motojima, head of the international fusion project, speaks out on delays, leaks and rumours.

\section{Elizabeth Gibney}

31 July 2014

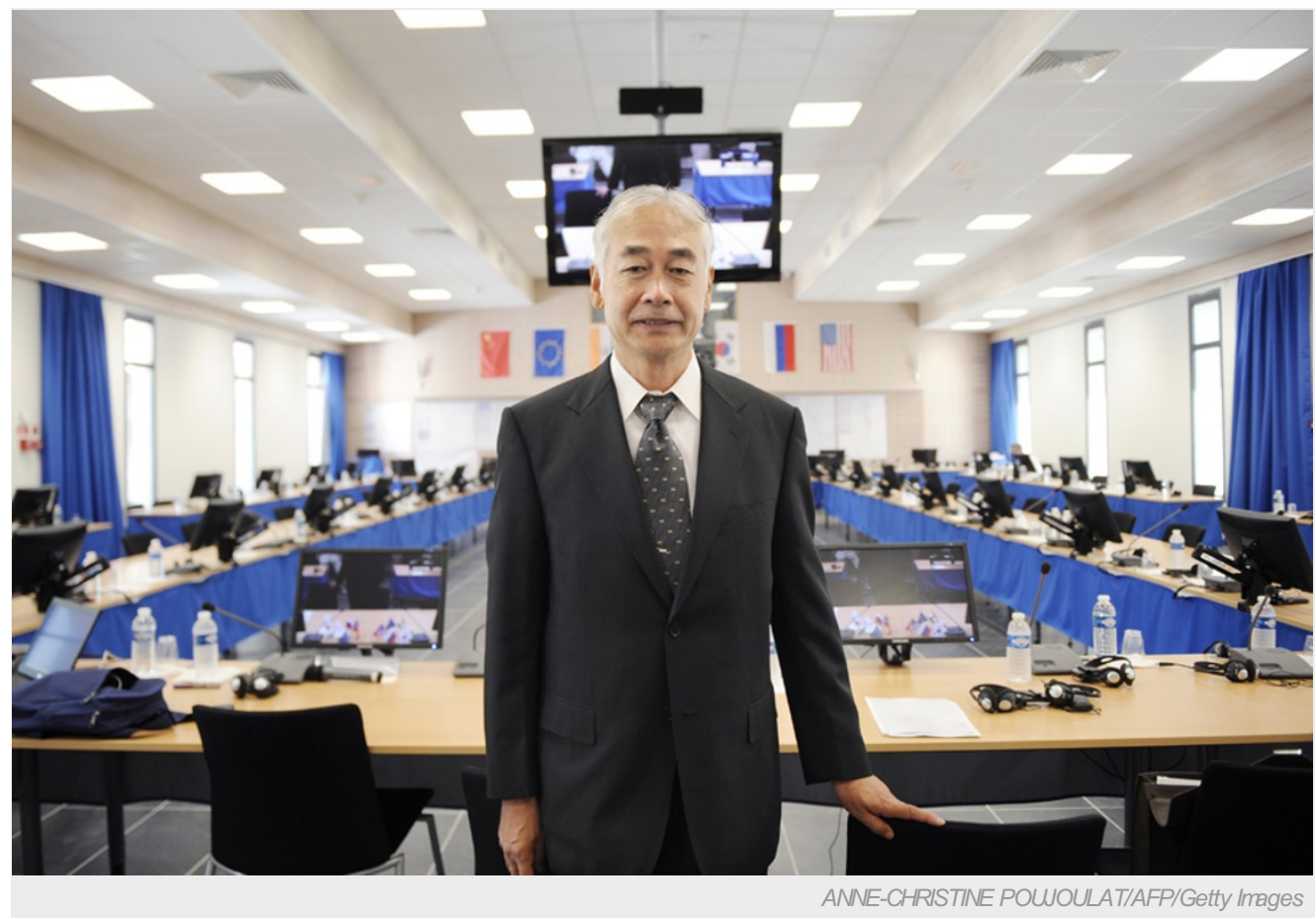

Osamu Motojima has led ITER since 2010.

Harnessing the power of the Sun on Earth has long been a dream for physicists. In 2006, a team from the European Union, China, India, Japan, South Korea, Russia and the United States got the go-ahead to realize that ambition by building an experimental reactor designed to use nuclear fusion to generate ten times the power that is put in.

The multibillion-euro ITER facility is now being built in St-Paul-lez-Durance, southern France. But after eight years, it has become complex and unwieldy. Following an extensive design review in 2009, ITER's projected costs trebled to around $€ 15$ billion (US $\$ 20$ billion), and construction delays - as well as unforeseeable events such as the 2011 Japanese earthquake, which damaged component-testing facilities - have meant that the research programme is now likely to be pared back.

Last year, a management assessment leaked by The New Yorker magazine (see go.nature.com/7ayyqe) gave a searing account of the organization's failures. Finding that the project was "in a malaise and could drift out of control", it recommended 11 urgent reforms. As construction of ITER's main experimental facility gets underway, outgoing director-general Osamu Motojima spoke to Nature about the report, delays and ITER's future.

The expected date of the first experiments confining super-hot plasma in ITER's doughnut-shaped magnetic chamber, or tokamak, has been pushed back from 2016 to 2020. At this year's meeting of the ITER Council in June, members said that the schedule needed to be updated, and stressed the need to stem slippage. What might a realistic schedule look like? Construction is quite difficult, because a lot of integration of parts is necessary. We are trying to estimate the date of the machine's completion and of first plasma, but it's a bottom-up process that integrates technical data and work schedules for different components made by different countries.

\section{Many people are openly talking about 2022 or 2023.}

There are a lot of rumours. I have the target date, but I need to demonstrate that we can do it with a high-enough probability. It will be around 2022 or 2023 and I will report to the ITER council next June. If the date is 2025, the project will never survive. That I know clearly. But it is important to remember that the success of ITER is not demonstrated with the first plasma, but when the deuterium- 
tritium plasma operation is realized — currently scheduled for 2027.

Last month, the US Senate proposed a budget that would see the United States pull out of ITER. How concerned are you? The United States has high scientific and technological capability in fusion energy and has been one of the leading countries in ITER from the beginning, so I have a strong concern about this US funding issue. I do not want to believe that the United States would pull out of ITER, because it would be a disaster to the project. However, I also believe the country might well recognize the advantage and benefit to staying in the project.

I would like to mention one important point. According to the ITER treaty, a member cannot withdraw before 2017 — and even if they do, their in-kind contribution to the construction is due. So this is more an internal problem in the United States.

The US House of Representatives' version of the budget proposes increased funding, as long as ITER implements the recommendations of last year's management report. How are you responding to those recommendations?

I accepted the need to address performance and make improvements, and therefore agreed to the 11 recommendations and to 31 actions. There are important actions, such as creating a 'project culture'. I first mentioned this to my staff in 2010, and now, relying on the recommendations, I am pushing very strongly to accelerate the process. Another very important thing is to show a strong nuclearsafety culture, which I have tried to encourage. We reported improvements to the ITER council last month.

But can I say a point to you frankly? We have a big problem. The ITER council determined that the 2013 management assessment, including its executive summary and recommendations, should be a confidential document. So although I am trying to be transparent and explain the 31-point action plan, I am not comfortable talking about this in detail in public.

\section{Would you have preferred that the report had not been leaked?}

Yes. I regret that this executive summary was uploaded to websites. This makes some things difficult and has caused a lot of unnecessary rumours.

The report said that ITER leadership was "not meeting the challenge", and one of the recommendations was to accelerate the transition to a new director-general. This seems to imply that the assessors laid some blame with you. Do you think this is fair?

No one ever said to replace me. I have the contract for five years and already four years have passed, so with or without the recommendation, I need to ask the ITER council to settle on the selection process for the new director-general.

\section{But do you think it is fair to blame ITER's shortcomings on you?}

That is an issue of the ITER council. If, one year before the process to determine the next director-general, they say that an acceleration of the selection process is necessary, it is not because the director-general is condemned. Right now, that is all that I can answer. Given that I am accepting this recommendation, I should not mention my personal feelings to you. One year from now, perhaps I can say more.

The report identified some wide-ranging problems, such as the complex relationship between the seven ITER members and the central organization. Are these problems that can be fixed, or are they too deeply entrenched?

About two years ago, I created the Unique ITER team with the ITER organization and seven domestic agencies to tackle this problem. The situation has improved a lot, but you are right: the problem comes from the basic design, that ITER is an international project. We are working hard every day — we want each member to maximize their benefit while cooperating. But some parts of ITER's structure that make it complex are important to fulfilling the project's other big objective: that all the intellectual property obtained is available equally to all seven members.

ITER seems to dominate fusion, mostly because of the enormous amount of money going into it. How important is it to test other fusion methods?

Tokamaks are now the most advanced plasma-confinement system, which is why this design was selected for the experimental reactor. I have confidence that we will contribute to DEMO [the Demonstration Power Plant, a proposed follow-up to ITER]. But there are other concepts that are also important, such as laser fusion and stellarator reactors, including the Wendelstein 7-X reactor in Germany and the Large Helical Device in Japan. National projects are important, because how do you move to the next stage of demonstration if you don't have any researchers or engineers in your country? In the United Kingdom, maybe 50 people work on ITER. Each country cannot contribute to the next stage of fusion with only 50 people. So to concentrate too much on ITER is dangerous. I say that even as its director-general. 
The crisis in Ukraine meant that you had to move this year's council meeting from St Petersburg to the ITER headquarters. Are you worried that international tensions could further affect ITER?

Yes, I have some concerns. It is quite dangerous for our project. But ITER is a scientific project aiming at a peaceful demonstration of fusion energy, and I wish it to be outside the tensions. That is why the council took the decision to move the meeting. I believe that all the members, including from the United States and Russia, also agreed that ITER should be beyond international tensions — not directly, but by sitting down together at the table.

Do you have a plan in case problems escalate?

In such a case, we are preparing a plan to mitigate the impact. We always manage to find the solution — that is the basic philosophy of the ITER project.

Nature | doi:10.1038/nature.2014.15621 